\title{
PRÁCTICAS MORTUORIAS WARI EN MARAYNIYOQ, VALLE DE AYACUCHO, PERÚ
}

\author{
WARI MORTUARY PRACTICES AT MARAYNIYOQ, \\ AYACUCHO VALLEY, PERU
}

\author{
Lidio M. Valdez ${ }^{1}$, Jocelyn S. Williams ${ }^{2}$ y Katrina J. Bettcher ${ }^{3}$
}

\begin{abstract}
Se presentan los resultados del análisis de los restos óseos humanos provenientes de dos estructuras mortuorias excavadas en el sitio Wari de Marayniyoq, del valle de Ayacucho, Perú. Hasta hace poco, las formas de enterramiento Wari fueron discutidas sólo a partir de las estructuras funerarias, prestándose escasa atención a los huesos humanos. Nuestros resultados indican la presencia de una población numerosa, con individuos de todas las edades y ambos sexos. Se concluye que durante el auge de Wari, diversas formas de enterramiento fueron practicadas en este valle; de estas, algunas parecen haber sido utilizadas por varias generaciones, resultando en la acumulación de abundantes restos óseos humanos al interior de estructuras mortuorias relativamente pequeñas. Finalmente, la evidencia proveniente de Marayniyoq se emplea para elaborar un perfil de la población allí depositada, la misma que deja en claro la ocurrencia de una alta mortalidad infantil.
\end{abstract}

Palabras claves: Andes Centrales, Wari, Ayacucho, entierros, antropología física.

Results are presented from the analysis of human remains excavated from two mortuary structures at the site of Wari of Marayniyoq in the Ayacucho Valley, Peru. Until very recently, Wari mortuary studies were aimed mostly at the analysis of the mortuary structures, paying little attention to the human remains. This study of osteological remains from two Wari mortuary structures revealed not only a large population lived at the site of Marayniyoq, but that the structures held individuals of all ages and both sexes. They conclude that during the Wari reign diverse forms of burial were practiced in the valley, and some were used for generations, resulting in an accumulation of numerous remains in the relatively small mortuary structures. Finally the evidence from Marayniyoq is used to elaborate on the population profile of the deposit, which clearly indicates a high infant mortality rate.

Key words: Central Andes, Wari, Ayacucho Valley, burials, physical anthropology.

En los Andes centrales, las sociedades que antecedieron a la cultura Inka y los mismos inkas (Pizarro 1965; Rowe 1946; Sillar 1992) mostraron gran preocupación por la muerte. Tal como aún sucede en la actualidad, también en el pasado la partida sin retorno de los seres queridos conllevó a una serie de procedimientos rituales, actos estos que a menudo eran sancionados por la sociedad de la cual formó parte la persona fallecida. Muchos aspectos de dichos rituales no han dejando muestras tangibles y recuperables por los arqueólogos. En consecuencia, para los especialistas, las pocas evidencias que se han mantenido, tal como las estructuras funerarias y sus contenidos, son de vital importancia para poder aproximarnos a las antiguas formas de disponer a los muertos por las diferentes culturas (Silverman 1996:5; Valdez et al. 2002a:389-390).

La cultura Wari, que se desarrolló teniendo como centro al valle de Ayacucho de la sierra central del Perú, ha sido interpretada como un estado que logró controlar gran parte de los Andes centrales durante el periodo conocido dentro de la literatura especializada como Horizonte Medio (Isbell 1988, 1997a; Menzel 1964; Schreiber 1992). Además de su ciudad capital, en el valle de Ayacucho, la administración Wari habría establecido muchos asentamientos, unos diferenciados de otros, refle-

\footnotetext{
Department of Anthropology, University of Victoria, PO Box 3050 STN CSC, Victoria, BC V8W 3P5 Canadá.1valdez@uvic.ca Department of Archaeology, University of Calgary. jswilliams90@ hotmail.com

2816 Shelbourne St. Victoria, BC Canadá. kjbettcher@yahoo.es
} 
jando así una jerarquía de sitios (Isbell y Schreiber 1978). La presencia fuera del valle de Ayacucho de una red de sitios planificados intrusivos, considerados como centros administrativos Wari, y artefactos afiliados con Wari, son algunos elementos diagnósticos visto por los especialistas como testimonio del control ejercido por el estado Wari sobre un territorio bastante amplio (Schreiber 1992).

La intención de este trabajo es dar a conocer nuevas evidencias con respecto a las prácticas funerarias Wari, con énfasis en el material óseo humano. La evidencia arqueológica proviene del sitio Wari de Marayniyoq (Valdez 2002, 2006; Valdez et al. 2002a, 2002b; Valdez et al. 2001, 2005), ubicado sólo a $4 \mathrm{~km}$ al norte de la ciudad capital Wari (Figura 1). Para poner en perspectiva la discusión, primero se hace referencia a las prácticas funerarias Wari conocidas para el valle de Ayacucho y luego se presenta la evidencia proveniente de $\mathrm{Ma}-$ rayniyoq. Teniendo como base dicha información, discutimos aspectos relacionados a la población Wari.

\section{Estudios Previos}

En los Andes centrales, el estudio de los patrones de enterramiento se concentran principalmente en la antropología física (Allison 1984; Blom et al. 1998; Carmichael 1988, 1995; Donnan y Mackey 1978; Donan y Cook 1997; Hampe 1982; Hoshower et al. 1995; Indriata y Buikstra 2001; Isbell 1997b; Isla 2001; Kent y Kowta 1994; Lozada y Buikstra 2002; Nelson y Castillo 1997; Stothert 1978; Verano 1995, 1997, 2001, 2003; Zapata 1997). Para el caso de Wari (ca. 550-1.000 d.C.) hasta hace poco no existían análisis que buscaran integrar estudios de antropología física y las formas mortuorias Wari (Tung 2003). Con dicha limitación, se hacía difícil discutir temas relacionados a estratificación social y género y era muy poco lo que se conocía acerca de las formas de enterramiento. Para el valle de Ayacucho las únicas evidencias con respecto a esta temática eran los reportes de Lumbreras (1974), Isbell et al. (1991) y Benavides (1991). Estos estudios no incluyeron análisis de los restos óseos provenientes de las estructuras mortuorias, lo que hacía difícil discutir aspectos relacionados a género y estatus. En consecuencia, no es una exageración sostener que discusiones con respecto a estratificación social han sido basadas sólo en observaciones arqueológicas.
Durante los últimos años, se ha dado un renovado interés en el estudio de las formas mortuorias Wari del valle de Ayacucho (Valdez et al. 2002a). Este interés surgió con el descubrimiento de una cámara funeraria en Seqllas, que, aunque disturbada, permitió la identificación de una estructura mortuoria definitivamente accesible (Bettcher et al. 1999; Valdez et al. 2000, 2002). No obstante el mal estado de preservación de los huesos, dicho hallazgo también permitió determinar que en tales estructuras se depositaron restos de varios individuos $(\mathrm{nmi}=16)$, de diferentes edades y probablemente de ambos sexos (Valdez et al. 2002a:396; Valdez et al. 2001:349, 2005:177). El posterior descubrimiento en Posoqoypata de tres cámaras funerarias adicionales (Valdez et al. 2002a:404; Valdez et al. 2001:346) cada una conteniendo los restos de varios individuos (por ejemplo una estructura contenía un mínimo de siete individuos) de diferentes edades (adultos y subadultos) puso a la luz que una práctica común Wari, por lo menos en el valle de Ayacucho, fue depositar los restos humanos en estructuras subterráneas dotadas de un acceso (Valdez et al. 2002a; Valdez et al. 2001, 2005). Esto implica que las estructuras podían ser abiertas y reabiertas para depositar nuevos restos, además de facilitar el contacto entre los vivos y los muertos. Los accesos de todas las estructuras estaban orientados hacia el este, lugar de las montañas sagradas (Wamani) de Rasuwillka (Valdez et al. 2001:350).

Junto a las cámaras funerarias también se descubrieron otras formas de enterramiento. Estas consisten de pequeñas estructuras de forma cilíndrica (cistas), excavadas directamente en el suelo natural y revestidas en piedra (Valdez et al. 2001:341). En cada una de estas estructuras se encontraron restos de un individuo, en posición sentada y con las extremidades inferiores flexionadas hacia el pecho (Valdez et al. 2002a:400). Estas estructuras contenían los restos tanto de adultos como de subadultos. Las estructuras disponían de un techo consistente en una laja lo suficientemente grande. Por cuanto esta laja estaba muy cerca a la superficie, éstas pudieron ser abiertas de tiempo en tiempo, tal vez en la misma forma como eran abiertas las cámaras funerarias, permitiendo de este modo el contacto de la población con el individuo fallecido.

Cistas cilíndricas han sido excavadas en el sitio Wari Muyo Orqo en Conchopata (Berrocal 


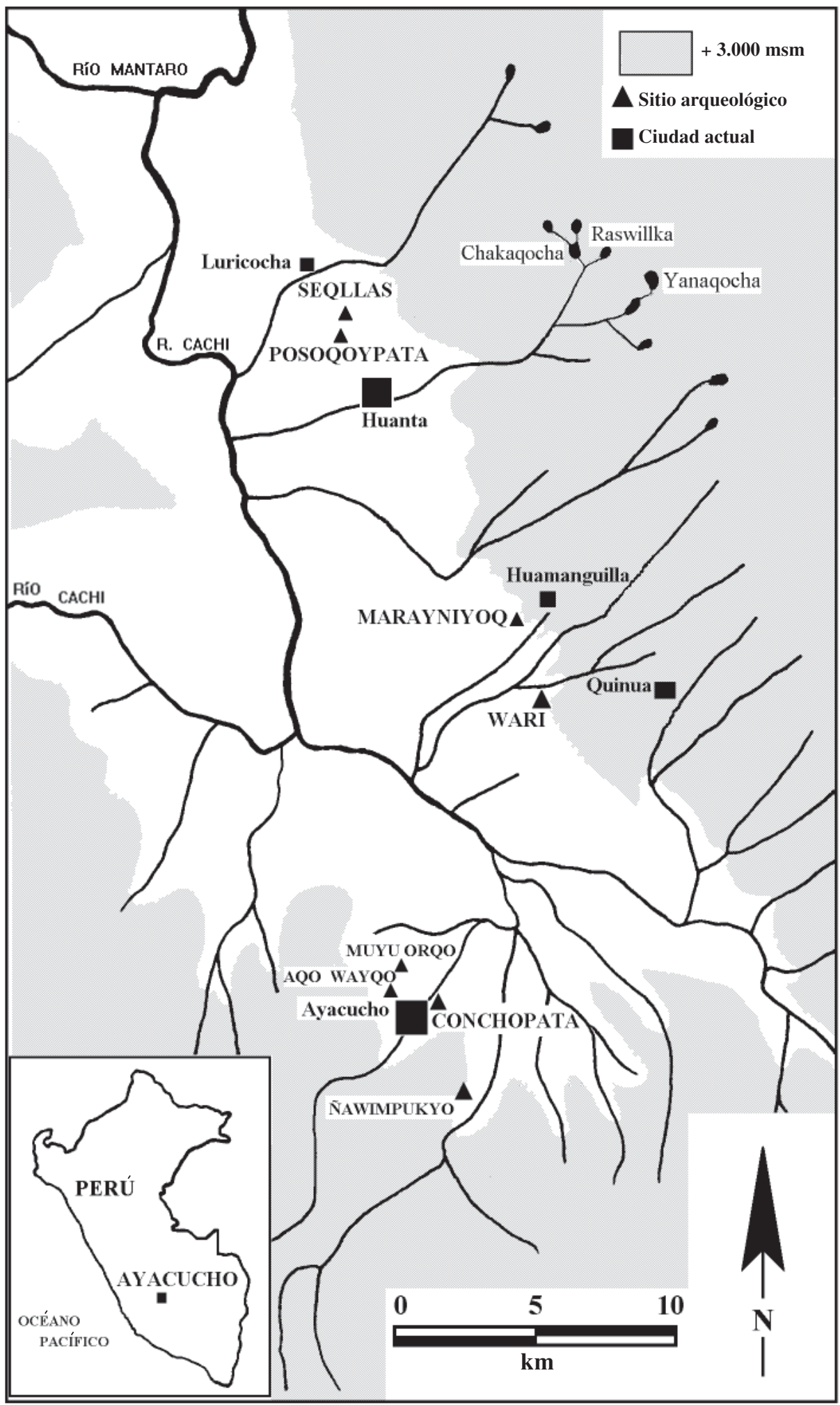

Figura 1. Ubicación del sitio arqueológico de Marayniyoq en el valle de Ayacucho. Location of the Marayniyoq archaeological site in the Ayacucho Valley. 
1991) y Ñawimpukyo (Machaca 1996:72); donde Ochatoma y Cabrera (2001) hallaron una tumba con doble cámara. En Conchopata también se han excavado varias estructuras funerarias, que incluyen simples fosas de forma cilíndrica, cistas también de forma cilíndrica y cistas en forma de bota (Ochatoma y Cabrera 2001) (J. Ochatoma, comunicación personal, 2004).

Isbell (2004) ha definido ocho formas de enterramiento para Conchopata. La primera forma consiste en una estructura cilíndrica excavada sobre el suelo natural, donde se depositaron los restos de un individuo en una posición sentada; la estructura carece de un revestido de piedra, pero sí contaba con una cubierta o techo consistente de una laja. La segunda forma es idéntica a la anterior, excepto que contenía dos individuos. La tercera forma es idéntica a la segunda de forma cilíndrica descubierta en Posoqoypata (Valdez et al. 2001:340-343); es decir, con paredes revestidas de piedras y un techo consistente de una laja (ver también Cook 2001:145). La cuarta forma consiste de "entierros profundos cavados en el lecho rocoso" (Isbell 2001:31) donde estaban depositados los restos de varios individuos, en posición sentada. La quinta forma se trata de un amplio ambiente, en cuyo interior estaban varias estructuras pequeñas de forma cilíndrica, con las paredes revestidas de piedra. De todas, una que parece ser la principal, es ligeramente más grande y contenía los restos de dos individuos, mientras las otras contenían sólo los restos de un individuo. Una forma más elaborada que la descrita en esta sección consiste de una cámara funeraria accesible y que contenía los restos de varios individuos (Isbell y Cook 2002:285-287), colocados siempre en una posición sentada, con las rodillas flexionadas hacia el pecho. Una sexta forma de enterramiento definida para Conchopata consiste de nichos que contenían los restos de dos individuos depositados manteniendo una posición sentada. Esta forma de enterramiento fue definida por Lumbreras (1974:180-181). Recientes excavaciones efectuadas en Posoqoypata por Ernesto Valdez en 2002 resultaron en el hallazgo de un entierro similar. La séptima forma de enterramiento definido para Conchopata consiste de una fosa ligeramente circular y donde se habían depositado los restos de cinco individuos. Esta forma de enterramiento fue definida en 1977 (Isbell y Cook 1987, 2002) y se sostiene que todos los individuos ahí depositados eran de sexo femenino y jóvenes (Isbell 2001:35).
Estas evidencias demuestran que durante el Horizonte Medio en el valle de Ayacucho había varias formas de enterramiento (Valdez et al. 2002a:404). Junto a todas estas formas estarían también las estructuras de Cheqo Wasi, interpretadas como lugares de enterramiento de la elite Wari (Benavides 1991; Isbell y Cook 2002). Además, Isbell especula que la estructura subterránea que tiene la forma de una llama, excavada por Ismael Pérez en la misma ciudad de Wari, fue el lugar de enterramiento del "Rey Wari” (Morell 2002:113), que a su vez representa la octava forma de enterramiento (Isbell 2004:20). El mencionado reporte, sin embargo, no da referencia al hallazgo de hueso humano alguno (Morell 2002).

Resumiendo, la información disponible muestra que la población Wari, por lo menos en su centro de origen, veneró a sus muertos en una forma bastante similar a la observada por los españoles para el caso Inka (Pizarro 1965:192; Rowe 1946:259). Aunque no hay evidencias de momificación de los cuerpos, se sabe que los muertos, o los huesos de los muertos, a menudo fueron guardados en estructuras accesibles, cuidadosamente construidas. Algunas de estas estructuras estaban situadas junto a los ambientes residenciales (Isbell y Cook 2002:282287), sugiriendo que los muertos y la población coexistieron, probablemente en mutuo contacto. En Cheqo Wasi (Wari) se observó un ambiente similar asociado a una cámara megalítica. Los estudios de rescate en Posoqoypata también expusieron un ambiente, asociado a una cámara funeraria accesible (Valdez et al. 2005).

\section{Las Estructuras Mortuorias de Marayniyoq}

Marayniyoq es un sitio Wari ubicado en la sección media del valle de Ayacucho (Valdez 2002, 2006; Valdez et al. 2000, 2002a, 2002b, 2005; Valdez et al. 2001). Las excavaciones realizadas entre 1999 y 2002 permitieron exponer dos estructuras funerarias de formas novedosas para el caso Wari, que se describen a continuación:

\section{La cámara}

Es una estructura rectangular subterránea, con techo y un acceso (Figura 2), nuevamente con orientación hacia las montañas de Rasuwillka. El techo, que estaba a escasos centímetros de la superficie, 


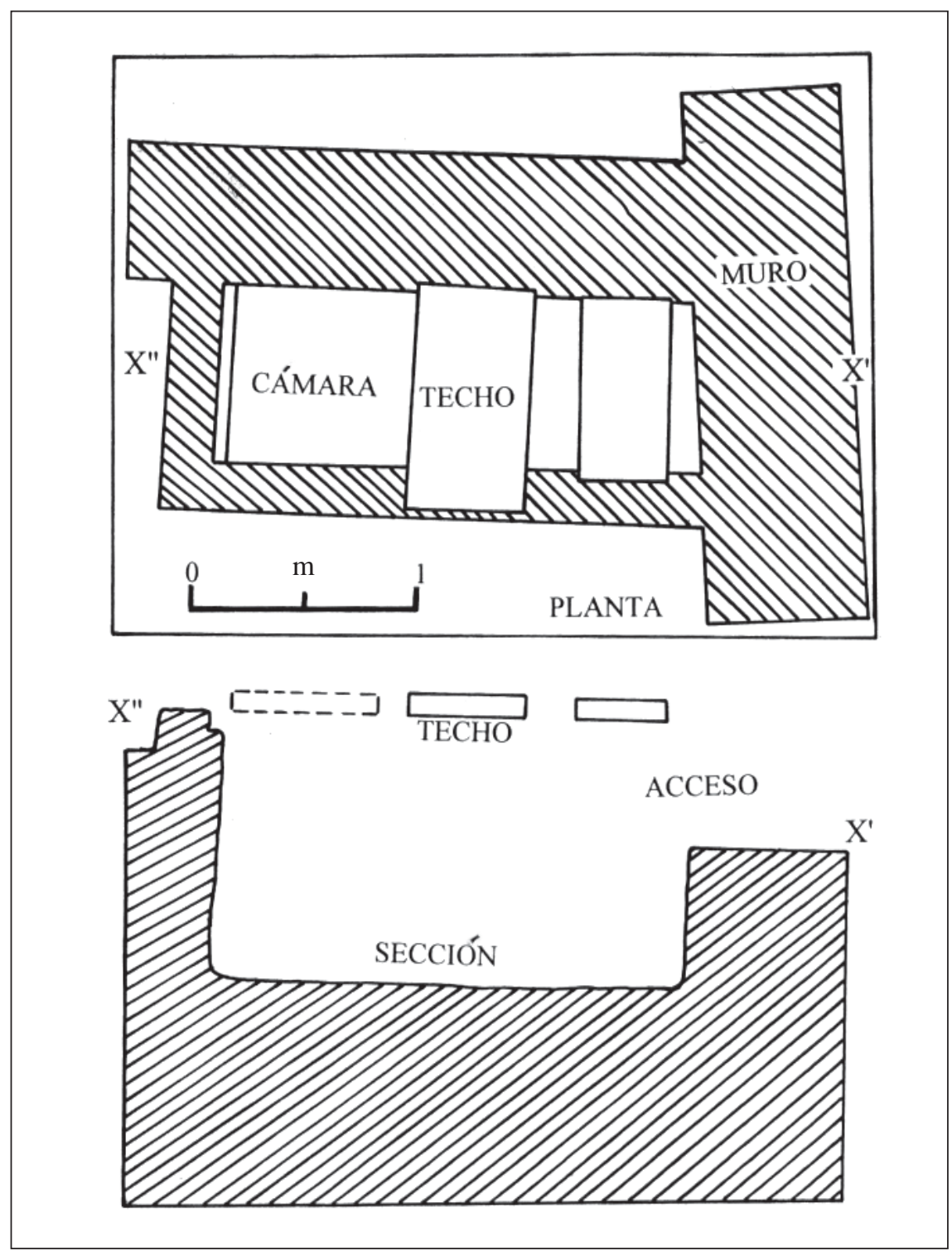

Figura 2. Dibujos de planta y sección de la cámara funeraria de Marayniyoq.

Drawings of the layout and a cross section of the funerary chamber from Marayniyoq.

consistía de dos bloques de piedras megalíticas, sobre las cuales se había colocado a modo de un sello una cubierta de arcilla bastante compacta. Una cubierta similar fue previamente observada para las cámaras subterráneas descubiertas en Seqllas y Posoqoypata (Valdez et al. 2002a:394). Las paredes de la estructura son de pirca y carecen de un enlucido. Las dimensiones de la cámara son: 2,08 $\mathrm{m}$ de largo, 0,80 $\mathrm{m}$ de ancho y una profundidad de $1,08 \mathrm{~m}$. Cerca a la esquina sureste se ubicó un pequeño nicho, similar al observado en Seqllas (Val- dez et al. 2000, 2002a), donde tal vez se depositaron las ofrendas. Por su lado norte pasa un largo muro en dirección este-oeste; el acceso de la cámara está conectado al referido muro. Es posible que, en su forma original, los muertos fueran orientados hacia las montañas de Rasuwillka.

La cámara había sido intervenida, resultando no sólo en el retiro casi total del ajuar funerario, sino también en la dispersión de las muestras óseas fuera de la estructura. El techo, en su lado sur, no tenía la cubierta de laja, simplemente estaba tapado 
con piedras de campo. Esto quiere decir que originalmente la estructura debió estar cubierta por tres piedras megalíticas. Entre las ofrendas halladas al interior de la cámara e inmediatamente fuera de la estructura, pero siempre asociadas a los huesos humanos, hay 10 miniaturas de cerámica de diferentes formas, pero de mal acabado y sin decoración (Valdez et al. 2005:181). Hallazgos similares han sido recuperados de otras estructuras funerarias Wari del valle de Ayacucho (Valdez et al. 2002b:39). Esta evidencia indica que las miniaturas formaron un aspecto sobresaliente de las ofrendas mortuorias Wari. Una pieza de Spondylus trabajada y cinco pequeños aros de oro, también son parte del ajuar funerario. Junto a los huesos, tanto al interior y fuera de la cámara, se hallaron huesos de cuy (Cavia porcellus), lo que sugiere que este roedor también sirvió como ofrenda. Indudablemente, esta relación no parece representar el total del ajuar funerario; por el contrario, es probable que cámaras funerarias, como la de Marayniyoq, contenían valiosas piezas, como indica el hallazgo de los aros de oro.

Muchas de las muestras óseas fueron halladas fuera de la cámara $(\mathrm{nmi}=48)$, en total desorden y desarticuladas. Otro grupo de huesos humanos habían sido dejados al interior de la cámara, pero siempre mezclados. Como resultado, queda incierto si originalmente los restos humanos estaban articulados y en orden. Es preciso anotar que algunos huesos de las extremidades inferiores fueron hallados aún articulados, flexionados, sugiriendo que originalmente los cuerpos fueron depositados en una posición sentada y con las rodillas flexionadas hacia el pecho.

\section{La fosa}

Esta estructura que contiene restos de varios individuos consiste de una fosa de forma rectangular y excavada en el suelo natural. De largo $(\mathrm{N}-\mathrm{S}$ ) mide $2,14 \mathrm{~m}$, de ancho $0,74 \mathrm{~m}$, con una profundidad que varía entre 0,50 y $0,32 \mathrm{~m}$ (Figura 3). En comparación a la cámara, esta estructura es bastante modesta. Carece de un techo, aunque sí estaba dotada de un piso pavimentado con lajas. En su interior se hallaron los restos desarticulados de varios individuos, todos acumulados sin orden alguno, lo que dificultó la separación de esqueletos completos. Sin embargo, en comparación a los huesos recuperados de la cámara previamente referida, los huesos de la fosa estaban mejor conservados. Aunque existe la posibilidad de que la fosa nunca fue saqueada, el ajuar funerario era bastante limitado, reflejando tal vez a los individuos allí depositados. Este consistía apenas de dos miniaturas de cerámica (Figura 4) bastante parecidas a las encontradas en la cámara.

Mientras que para el caso de la cámara se tiene alguna indicación que sugiere que los cuerpos parecen haber sido colocados en una posición sentada, los cuerpos depositados en la fosa definitivamente recibieron un tratamiento distinto. Todo pa-

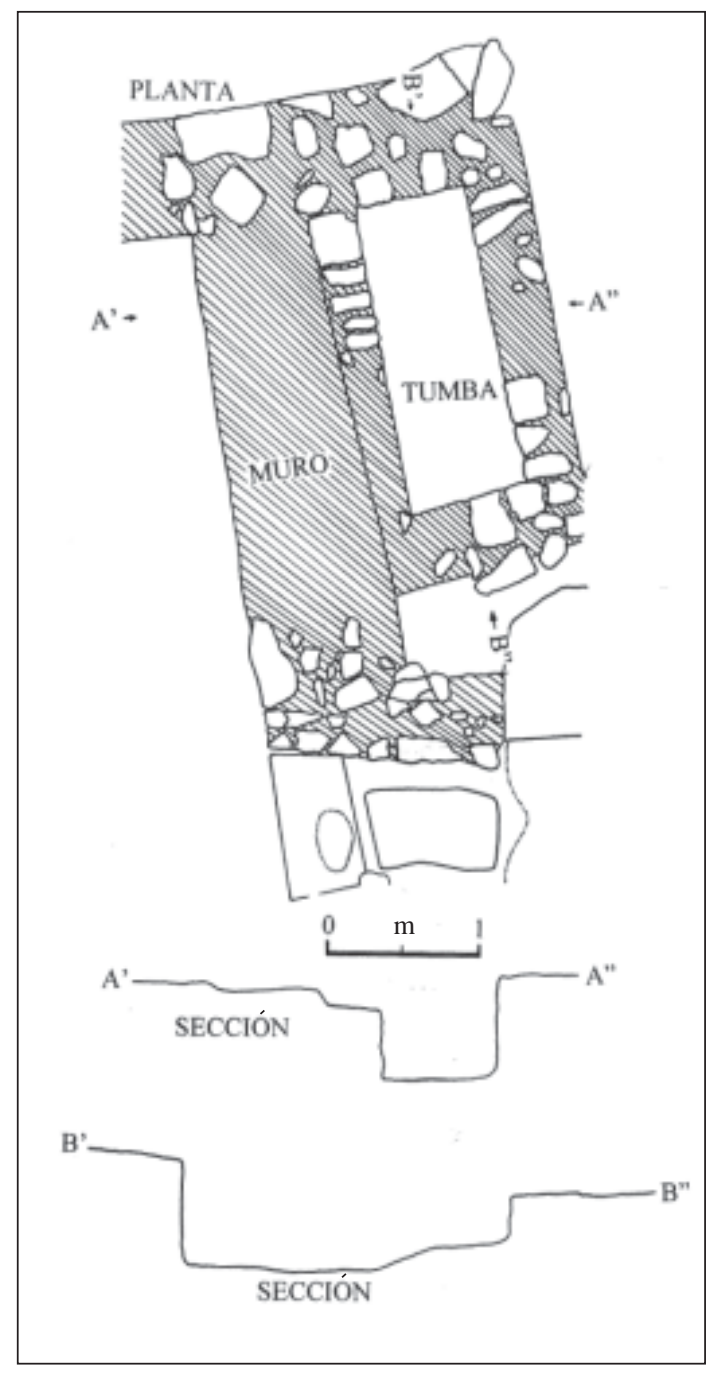

Figura 3. Dibujos de planta y sección de la fosa funeraria de Marayniyoq.

Drawings of the layout and a cross section of the grave from Marayniyoq. 
rece indicar que los huesos fueron trasladados de algún lugar, resultando en la mezcla total de los huesos, aunque cabe indicar que algunas vértebras aún estaban articuladas. Importante es añadir ade- más que entre los huesos de la costilla se halló un instrumento de hueso (Figura 5), bastante punteado (Figura 6), que parece haber penetrado a la altura del pecho y logró salir por la espalda.

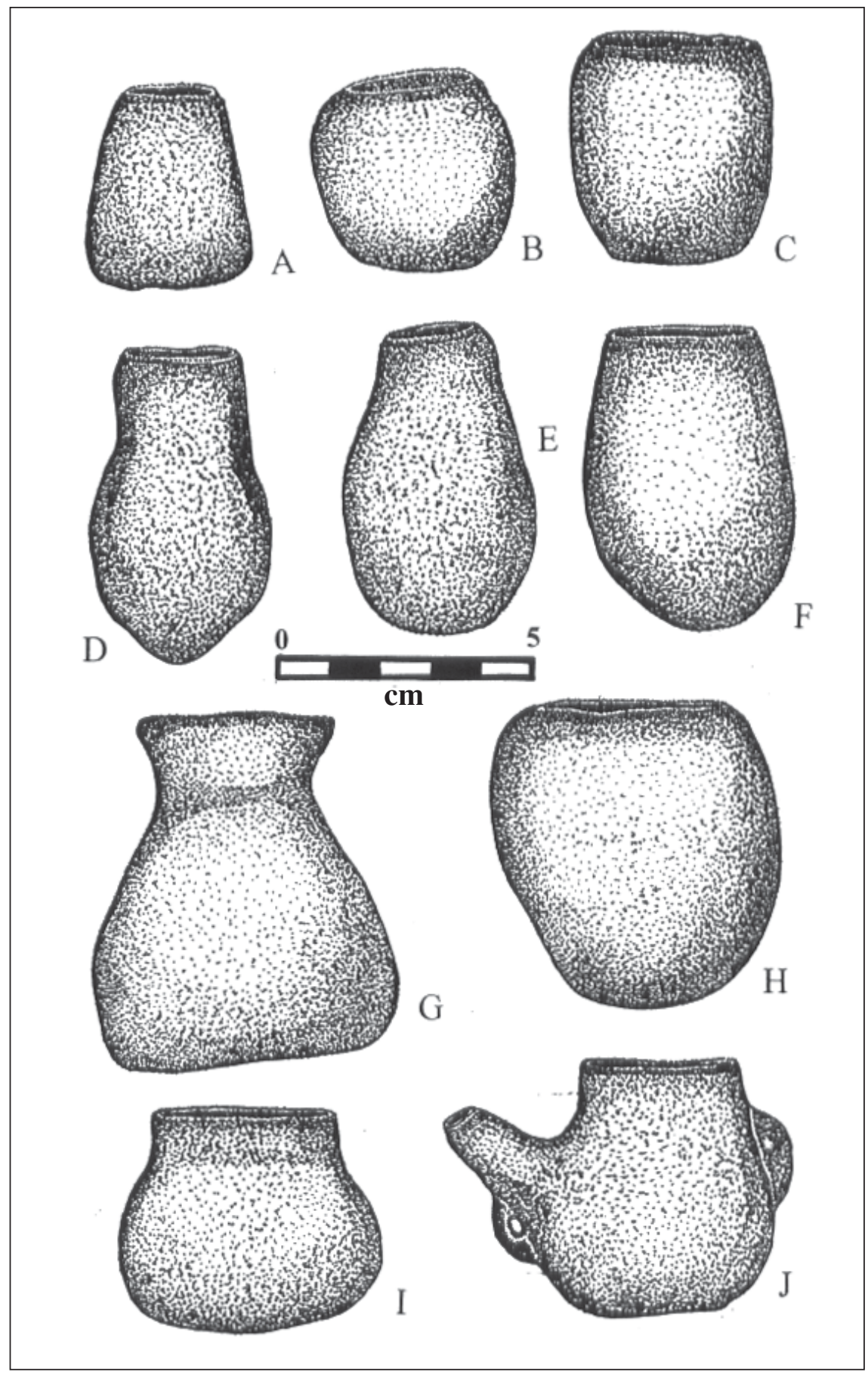

Figura 4. Miniaturas de cerámica asociadas a la fosa de Marayniyoq. Miniature ceramics associated with the grave from Marayniyoq. 


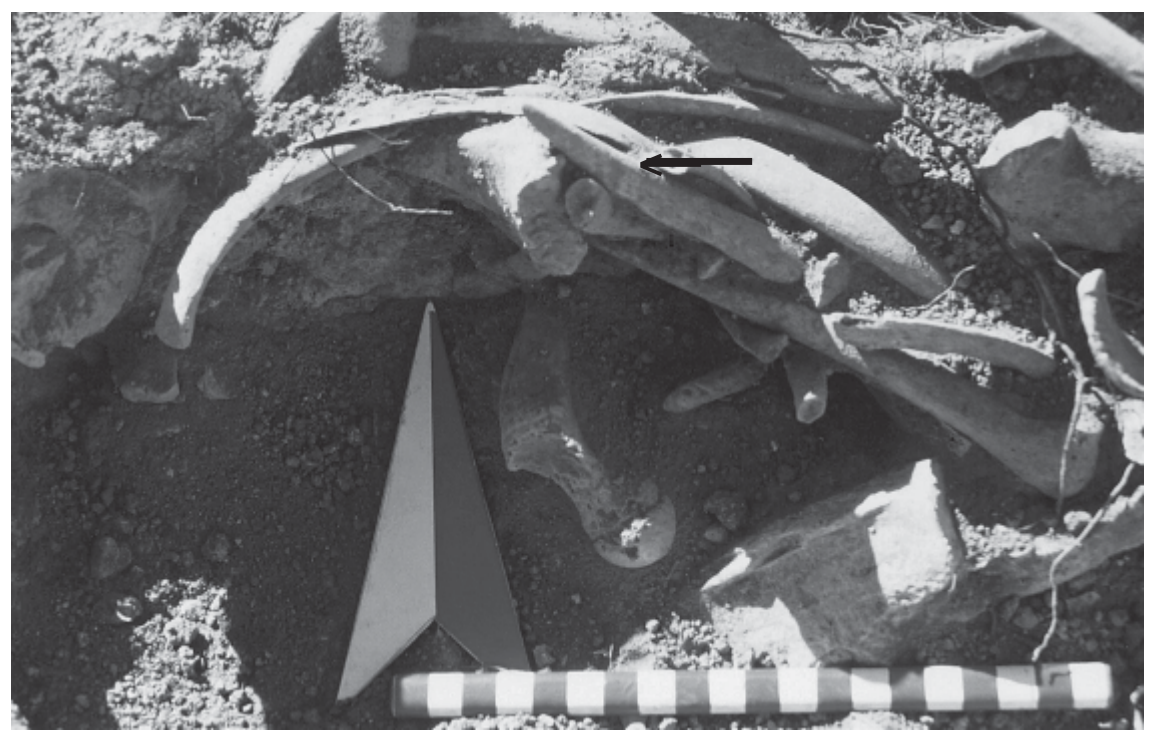

Figura 5. Instrumento punzante de hueso asociado a los huesos humanos de la fosa. Sharpened bone instrument associated with the human remains in the grave.

\section{Metodología}

La colección de huesos humanos encontrados fue limpiada y posteriormente analizada por separado con el principal objetivo de determinar el número mínimo de individuos (nmi) y reconstruir el perfil demográfico de los restos depositados en cada una de las estructuras, de acuerdo a los parámetros de Bass (1987), Ubelaker (1978) y Buikstra y Ubelaker (1994).

En primer lugar se procedió con la identificación y separación de los huesos en grupos distintos

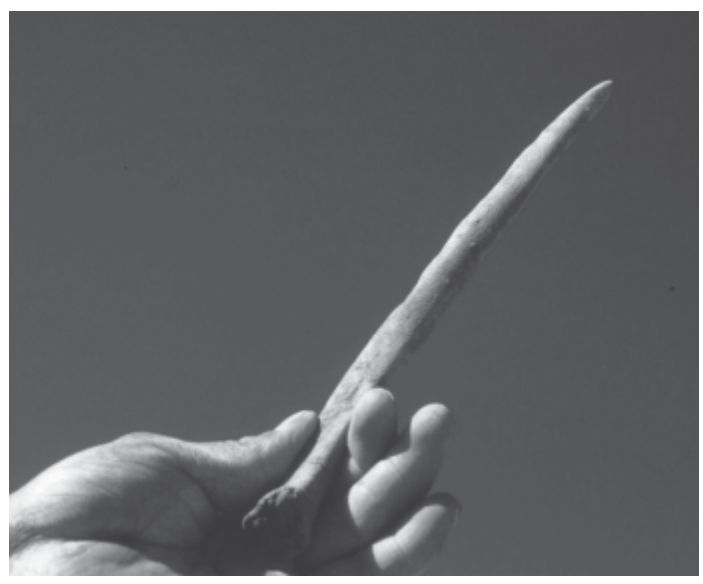

Figura 6. Detalle del instrumento punzante. Detail of the sharpened instrument. (p. ej. grupos de mandíbulas, grupos de tibia, grupos de fémur, etc.). Durante este procedimiento se realizaron observaciones relacionadas a patología, traumas, salud y modificaciones culturales (deformación craneana y trepanación). En seguida se procedió con la identificación de la edad, separando las muestras en dos grupos: subadultos (menores de 19 años) y adultos (mayores de 20 años). La estimación de la edad de este grupo se basó en el desarrollo dental (Ubelaker 1978:63), complementado con observaciones concernientes al tamaño de los huesos largos, la fusión de las epífisis, deterioro dentario, cierre de las suturas del cráneo y la morfología de la pelvis (Krogman e Iscan 1986). Cuando fue posible, los individuos subadultos fueron separados en neonatos/recién nacidos (0-3 años), niños (4-14 años) y adolescentes (15-19 años).

El siguiente paso fue el estudio de cada categoría (adulto, subadulto), evaluándose el lado derecho o izquierdo y, cuando fue posible, la edad y el sexo (Buikstra y Ubelaker 1994: anexo 2). Fichas adicionales se usaron para evaluar las mandíbulas y maxilares, siguiendo los criterios de Buikstra y Ubelaker (1994). Mediante este procedimiento se estableció en número mínimo de individuos (nmi). El sexo se determinó mediante la evaluación de la pelvis (Bass 1987:81; Buikstra y Ubelaker 1994). 


\section{Resultados}

\section{Cámara de Marayniyoq}

El análisis óseo de los restos provenientes de la cámara demuestra la presencia de una variada población, de todas las edades (Figura 7) y ambos sexos. No obstante que la muestra es pequeña $(\mathrm{nmi}=48)$, la distribución de la edad al tiempo de la muerte revela una baja mortalidad durante la infancia y una alta mortalidad durante la niñez (Figura 8). En el grupo de adolescentes, se observa un ligero declive de la mortalidad. Si comparamos este resultado con datos de Conchopata (Tung 2003:123), existen algunas diferencias, especialmente con respecto a la mortalidad durante la infancia y la niñez, que son bastante altas para el caso de Conchopata. Por su lado, la mortalidad entre los adolescentes de Conchopata es baja, pero relativamente alta entre aquellos cuyas edades oscilan entre los 20 y 34 años. En Marayniyoq, la mortalidad entre los adolescentes es segunda al grupo de niños y mayor que los del grupo cuyas edades oscilan entre los 20 y 34 años. En general, la distribución de la edad al tiempo de la muerte de los individuos provenientes de la cámara de Marayniyoq es distinta comparada a los datos provenientes de Conchopata.

Además, un mayor número de subadultos fue depositado al interior de la cámara (Figura 9), ya que constituyen el 58\%, comparado a un $42 \%$ de adultos. Para el caso de Conchopata, Tung (2003:122) no provee los datos en términos de adultos y subadultos, haciendo difícil hacer una comparación. En cuanto al sexo, había un mínimo de nueve individuos de sexo masculino (19\%), frente a siete de sexo femenino (15\%) (Figura 8). Como ya se anotó, un $66 \%$ están compuestos por subadultos cuyo sexo no fue posible determinar. Este resultado es diferente al de Conchopata, donde los de sexo femenino representan casi el doble de la población de sexo masculina (Tung 2003:124).

\section{Fosa de Marayniyoq}

El análisis de la colección ósea proveniente de la fosa de Marayniyoq también demuestra la presencia de varios individuos (Figura 10) y de ambos sexos. En primer lugar, la distribución de la edad al tiempo de la muerte (Figura 11) ilustra

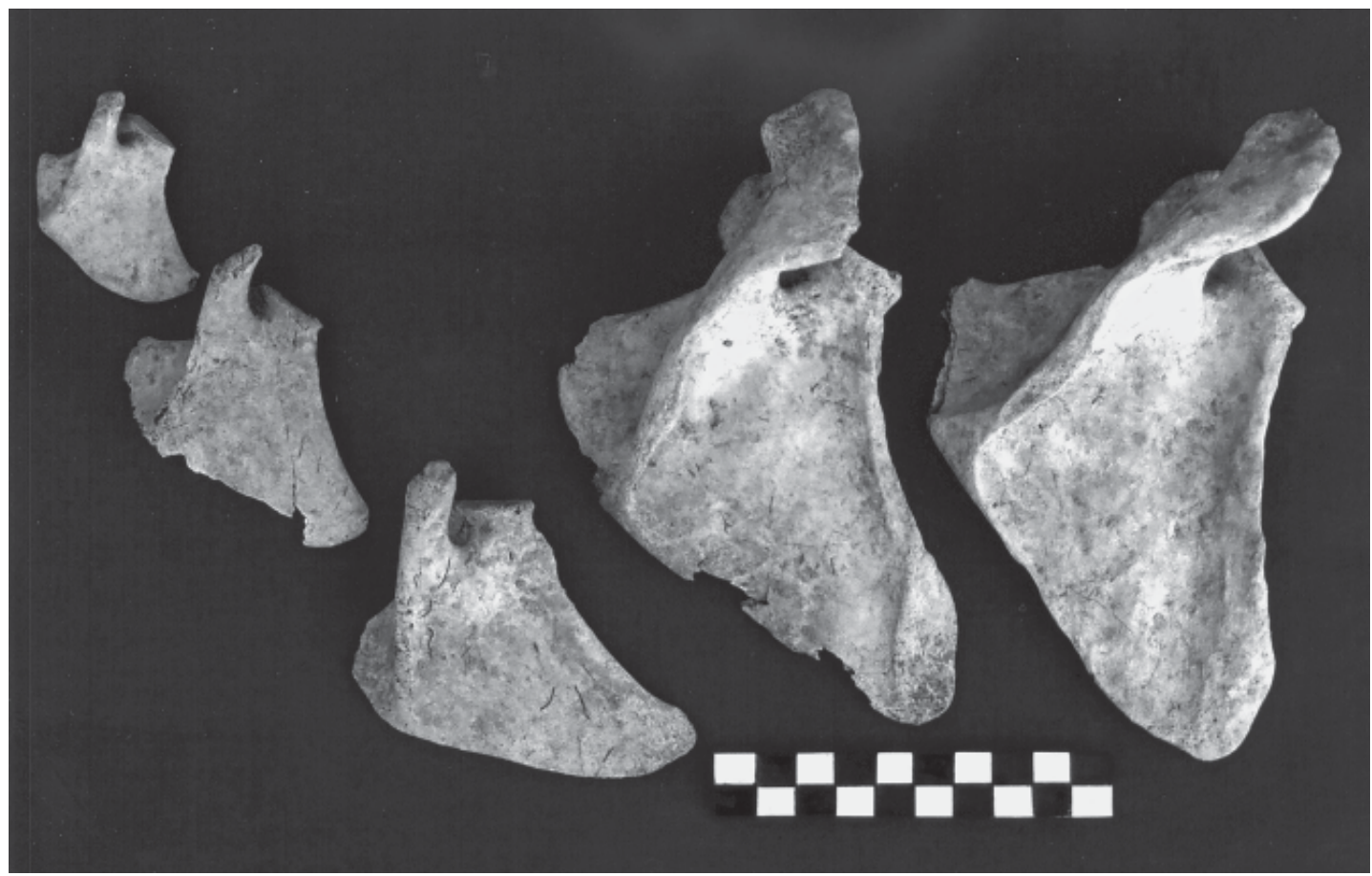

Figura 7. Escápulas pertenecientes a individuos de diferentes edades provenientes de la cámara funeraria, escala en $\mathrm{cm}$. Scapulae representing a variety of ages associated with the burial chamber, scale in $\mathrm{cm}$. 
nuevamente una alta mortalidad durante la niñez, la misma que es comparable a los restos de la cámara. Este resultado también es comparable a la información proveniente de Conchopata (Tung 2003:123). Al igual que para este último, donde hay menor ocurrencia de muertes entre los adolescentes, la información recuperada del análisis de los restos de la fosa también revela un caso parecido, pero distinto en comparación a los de la cámara. Nuevamente, existe un paralelismo entre los datos obtenidos para Conchopata y los de la fosa

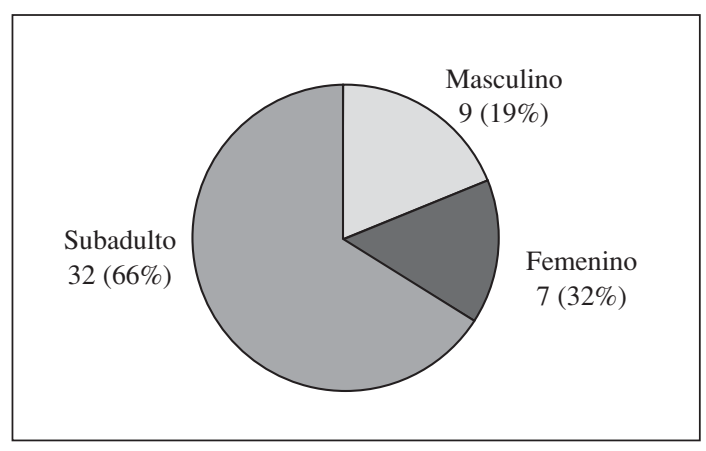

Figura 8. Distribución por sexo y edad (adultos y subadultos) de los individuos de la cámara.

Distribution by sex and age (adults and subadults) of individuals found in the chamber. con respecto a la mortalidad entre los individuos cuyas edades oscilan entre los 20 y 34 años, pero distinto en comparación a la información obtenida para los restos de la cámara. Como se podrá notar en la Figura 11, hay un grupo de adultos (mayores de 20 años) que probablemente pertenecen al grupo cuyas edades oscilan entre los 35 y 49 años, pero que la misma no fue posible confirmar durante los análisis. Si esta observación es correcta, es decir si el grupo de adultos pertenece al grupo arriba indicado, el declive en cuanto a mortalidad se refiere es ligero, habiendo pocas muertes entre los adultos mayores de 50 años. Esto, al igual que para la cámara, obviamente se debe a que pocos individuos sobrevivieron hasta dicha edad.

En contraste a los resultados presentados en la Figura 9, donde se observa la presencia de un mayor número de infantes, los resultados obtenidos para los restos de la fosa indican una distribución muy parecida entre adultos $(50 \%)$ y subadultos $(50 \%)$ (Figura 11). Por su parte, en cuanto a sexo se refiere, existen algunas dificultades, por cuanto no fue posible determinar el sexo de todos los individuos adultos con cierta precisión. Como se observa en la Figura 12, hay un grupo de población adulta, que representa un $19 \%$, cuyo sexo no fue posible determinar.

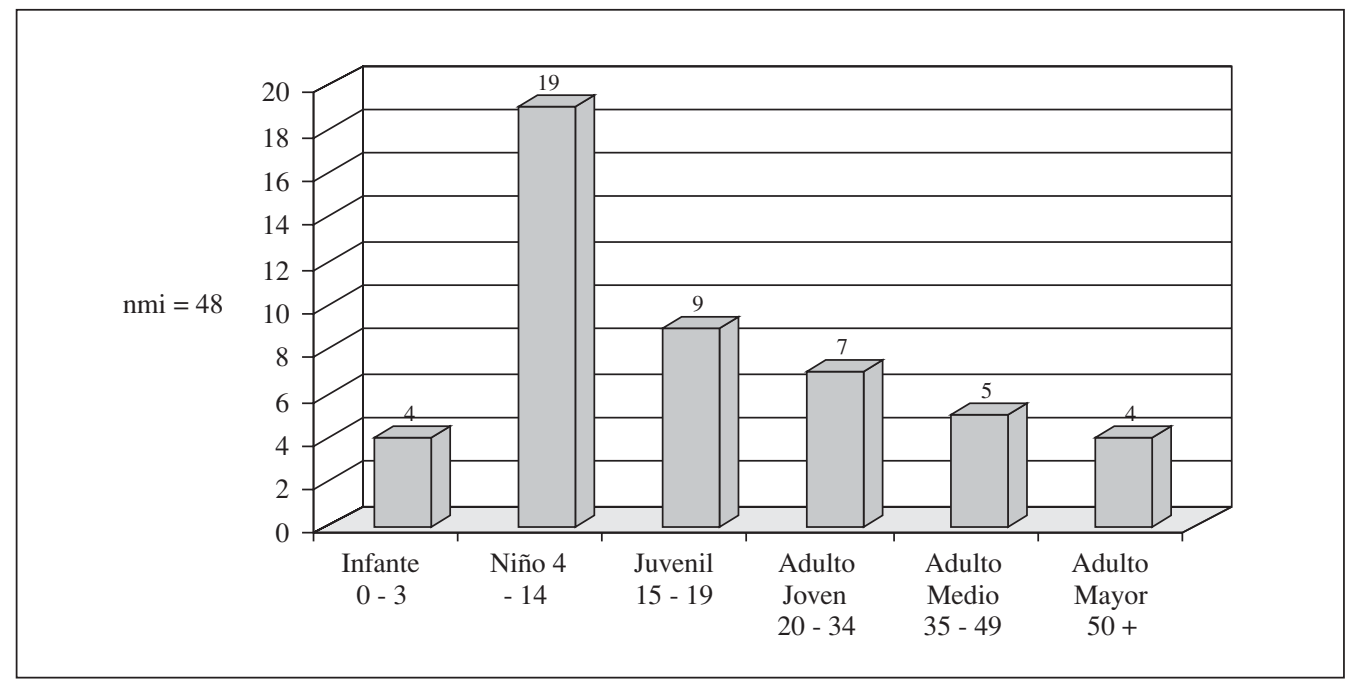

Figura 9. Distribución de la edad al tiempo de la muerte de los individuos depositados al interior de la cámara de Marayniyoq $(\mathrm{n}=48)$. La barra vertical representa el $\mathrm{nmi}$ (número mínimo de individuos).

Distribution of age at death of the individuals $(n=48)$ from the Marayniyoq chamber. The vertical bar represents the mni (minimum number of individuals). 


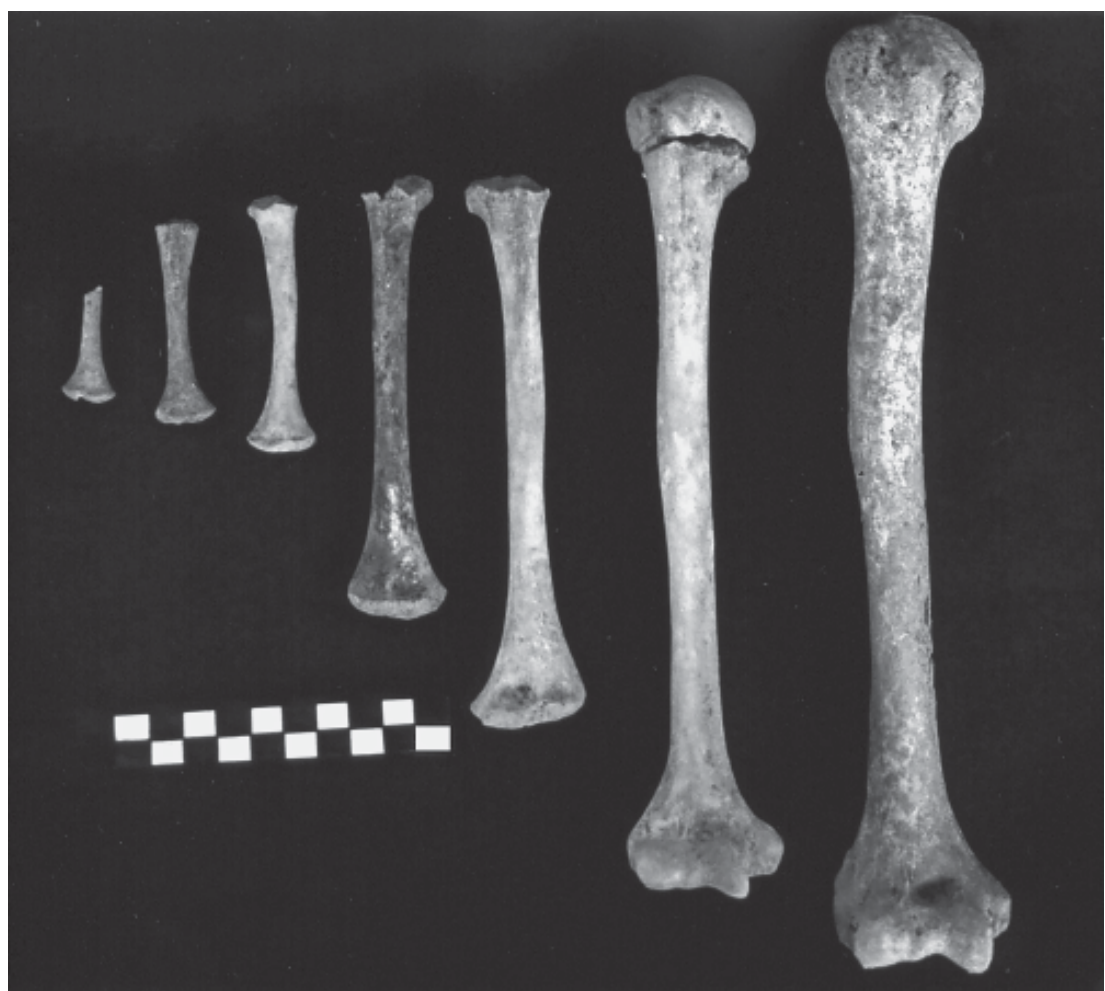

Figura 10. Húmeros pertenecientes a individuos de diferentes edades provenientes de la fosa, escala en $\mathrm{cm}$.

Humeri representing a variety of ages associated with the grave, scale in $\mathrm{cm}$.

\section{Discusión y Conclusiones}

La información extraída del estudio de los huesos humanos de Marayniyoq muestra una práctica relativamente común de la población Wari del valle de Ayacucho, la misma que consistió en mante-

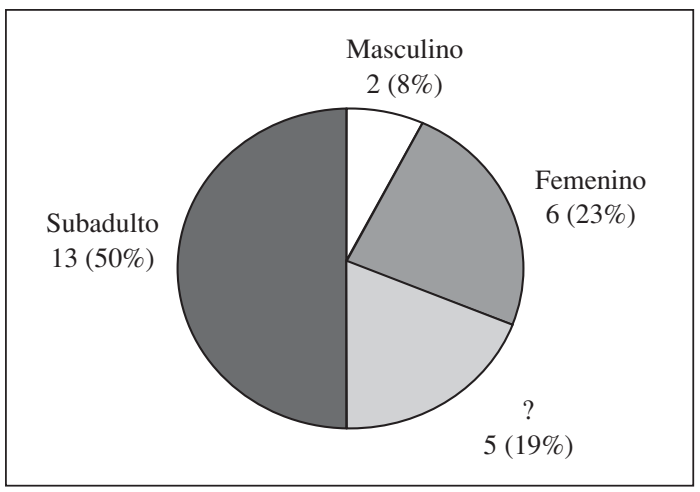

Figura 11. Distribución por sexo y categorías (adulto y subadulto) de los restos depositados en la fosa.

Distribution by sex and age (adult and subadult) of the remains deposited in the grave. ner juntos los restos, o los huesos, de varios individuos en estructuras funerarias construidas para tal propósito. Para facilitar aquella práctica, fue indispensable -en muchos casos- construir estructuras mortuorias accesibles que fueron utilizadas por varias generaciones (Isbell y Cook 2002:285; Valdez et al. 2002:404). La presencia de un mínimo de 48 individuos al interior de una estructura relativamente pequeña precisamente apunta en dicha dirección. Los cuerpos parecen haber sido depositados por varias generaciones, por lo que aquellos individuos introducidos con anterioridad (y como tales descompuestos y desarticulados) posiblemente fueron fácilmente arrimados para dar cabida a nuevos cuerpos. El perfil de edad al tiempo de la muerte de la población depositada en la cámara (Figura 9) indicaría esta posibilidad.

Por su lado, la fosa era más modesta en comparación a la cámara y carecía de acceso; su construcción obviamente también requirió poco esfuerzo. A diferencia de la cámara, los restos depositados en la fosa parecen constituir resultado de un evento único. Efectivamente, la presencia de un instru- 


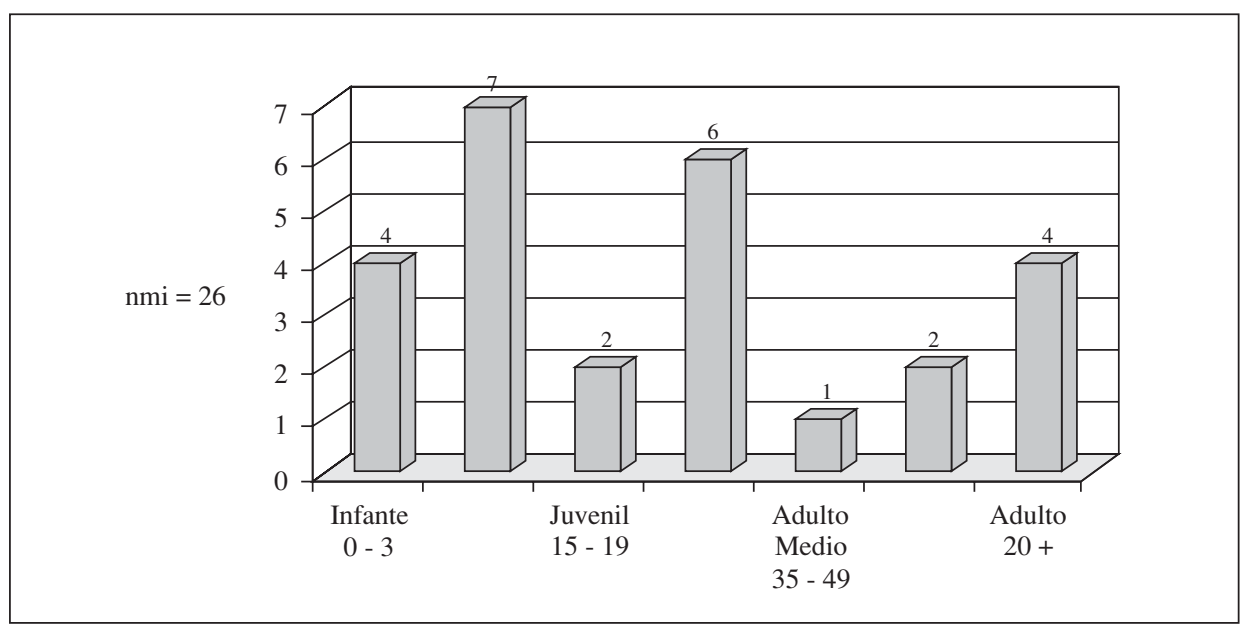

Figura 12. Distribución de la edad al tiempo de la muerte de los individuos depositados al interior de la fosa de Marayniyoq $(\mathrm{n}=26)$. La barra vertical representa el nmi (número mínimo de individuos).

Distribution of age at death of the individuals $(n=26)$ from the Marayniyoq grave. The vertical bar represents the mni (minimum number of individuals).

mento punzante junto a los restos de uno de los individuos hallados en la fosa, da la posibilidad de sugerir, como ocurre con la séptima forma de enterramiento discutido por Isbell (2001:31), que esta fosa fue producto de un sacrificio masivo. Apoya esta idea el hecho de que varios de los esqueletos aún estaban articulados. El perfil de edad al tiempo de la muerte de la población depositada en la fosa (Figura 12) sugiere un comportamiento distinto al observado para la cámara.

De los datos aquí presentados y la breve referencia dada a las diversas formas de estructuras funerarias Wari descubiertas últimamente en el valle de Ayacucho, también queda evidente que durante el tiempo de auge de la cultura Wari existieron diversas formas de enterramiento en este valle (Isbell 2004; Isbell y Cook 2002; Ochatoma y Cabrera 2001; Valdez et al. 2001, 2002a, 2005). Un buen número de estos contenían los restos de múltiples individuos. Por ejemplo, una estructura excavada en Conchopata, un centro especializado en la producción de la cerámica (Pozzi-Escot 1985, 1991), contenía los restos de un adulto de sexo masculino, junto a los restos de siete individuos de sexo femenino y varios subadultos (Isbell y Cook 2002:284).

Con base a la evidencia proveniente de Conchopata, Isbell (2004; Isbell y Cook 2002:287) postula que los patrones de enterramiento Wari reflejan la existencia de una "diferenciación social."
Los nobles y hombres de alta jerarquía social habrían recibido mejor trato y a menudo enterrados en compañía de sus varias mujeres (poligamia) e hijos. En contraste con las interpretaciones de Isbell (2001, 2004), Tung (2003:286) sostiene que la menor ocurrencia de los restos de individuos de sexo masculino en sitios como Conchopata podría ser resultado de otras actividades, como es el caso del mayor envolvimiento de la población masculina en proyectos estatales llevados a efecto en lugares lejanos.

Es oportuno anotar que existen notables diferencias entre los datos provenientes de Conchopata (Tung 2003) y Marayniyoq. En la cámara de Marayniyoq la mayoría de los restos pertenecen a subadultos, mientras la distribución entre la población femenina y masculina es bastante parecida. Es decir, no hay mayor presencia de individuos de sexo femenino tal como ocurre en Conchopata (Tung 2003). El caso de la fosa es distinto en relación a la cámara, pero bastante parecido a los casos reportados de Conchopata. Por lo tanto, la limitada información de que disponemos viene demostrando la existencia de diferencias significativas con respecto a la forma de enterrar a los muertos, cuyo significado (o significados) sigue siendo difícil comprender. Además las diferencias entre los resultados provenientes de Marayniyoq y Conchopata no permiten, por el momento, hacer generalizaciones sobre toda la población Wari. 
Por lo tanto, es evidente que las prácticas mortuorias Wari en el valle de Ayacucho son más complejas que la clasificación "jerárquica" basada exclusivamente en la forma de las estructuras mortuorias. Lo único definido hasta ahora es la considerable variación en la forma de estas estructuras mortuorias, pero cuyas implicancias aún son desconocidas. Al mismo tiempo, su contenido también es heterogéneo. Si bien el concepto general fue construir estructuras a modo de viviendas subterráneas, unas eran más amplias que otras, y algunas mejor acabadas en relación a otras. Algunas contenían los restos varios individuos, mientras que otras estaban casi vacías, indicando tal vez que tales estructuras fueron abandonadas siguiendo una probable reubicación de la población que las edificó.

Algunas comunidades parecen no sólo haber abierto las estructuras funerarias, estableciendo de este modo un contacto periódico con los muertos, sino también parecen haber cohabitado con los muertos en las mismas estructuras. Efectivamente, una estructura excavada en Posoqoypata reveló la presencia de un ambiente donde dos estructuras rectangulares y conectadas entre sí habían sido ocupadas simultáneamente. En la parte central de la estructura ubicada al lado Este se descubrió una laja colocada a nivel del piso. Una vez retirada la laja se determinó que cubría el acceso de una cámara construida por debajo del piso del cuarto ubicado al lado oeste. La estructura era larga y el techo consistía de un total de siete grandes lajas de piedra; sobre éstas se había colocado un sello de arcilla que no sólo permitió cubrir la estructura, sino también formó el piso del cuarto. En la cámara, bastante amplia, estaban los restos de dos individuos, pero en muy pobre estado de conservación (Valdez et al. 2005:184-185). Entre el ajuar funerario se encontró una vasija cara gollete representando a un individuo de sexo masculino llevando un poncho. Este ejemplo ilustra que muchas de las estructuras mortuorias Wari no sólo eran accesibles, sino que los vivos parecen haber buscado las mejores formas de poder coexistir con los muertos.

En general, los resultados provenientes de Marayniyoq demuestran la presencia de una alta mortalidad entre la población subadulta, especial- mente en el grupo cuyas edades varían entre los 4 y 14 años de edad (Figura 9). Luego, el declive es uniforme, lo cual indica que por lo menos los restos depositados al interior de la cámara representan muertes naturales. El menor número de muertos entre la población adulta obedece a que pocos individuos llegaron hasta dicha edad. Tal como se ilustra en la Figura 12, este no es el caso con los restos provenientes de la fosa, lo cual deja abierta la posibilidad que los restos allí depositados no parecen representar muertes naturales. Tung (2003:123) presenta los resultados de Conchopata y también observa la ocurrencia de una alta mortalidad entre la población cuyas edades oscilaron entre los 4 y 14 años. Luego, al igual que para la cámara de Marayniyoq, Tung (2003) observa una menor ocurrencia de muertes entre la población cuyas edades oscilan entre los 15 y 19 años. Estos son datos nuevos, pero para sacar conclusiones generales se hace necesario obtener datos similares de otros sitios Wari de la región. La recurrencia de comportamientos similares desde luego permitirá elaborar planteamientos más conclusivos, pero que a la fecha estamos lejos de contar con resultados comparativos.

Agradecimientos: Extendemos nuestra gratitud a todas las personas que participaron durante las varias temporadas de campo del Proyecto Arqueológico Marayniyoq; también a las autoridades del Instituto Nacional de Cultura del Perú por haber cedido los respectivos permisos para llevar adelante las excavaciones. Nuestra gratitud a los vecinos del sitio arqueológico por el interés mostrado en nuestro trabajo y sobre todo por la colaboración ofrecida. Brain Finucane (Oxford University) completó el estudio osteológico y gentilmente proporcionó información con respecto al nmi, edad y sexo de las colecciones aquí discutidas. Los trabajos en Marayniyoq fueron posibles gracias a una beca otorgada al primer autor por la Social Sciences and Humanities Research Council de Canadá, institución a la cual extendemos nuestro agradecimiento. Finalmente, nuestro reconocimiento a los varios evaluadores y editores de Chungara por sus críticas y comentarios a las versiones iniciales de este trabajo. 


\section{Referencias Citadas}

Allison, M.J.

1984 Paleopathology in Peruvian and Chilean populations. En Paleopathology and the Origins of Agriculture, editado por M.N. Cohen y G.J. Armelagos, pp. 515-529. Academic Press, Orlando.

Bass, W.M.

1987 Human Osteology: A Laboratory and Field Manual. Missouri Archaeological Society, Columbia.

Benavides, $\mathrm{M}$.

1991 Cheqo Wasi, Huari. En Huari Administrative Structure: Prehistoric Monumental Architecture and State Government, editado por W.H. Isbell y G.F. McEwan, pp. 55-69. Dumbarton Oaks, Washington, D.C.

Berrocal, M.

1991 Estudio Arqueológico en Muyи Orqo. Informe presentado para optar el grado de Bachiller en Arqueología. Facultad de Ciencias Sociales, Universidad de Huamanga, Ayacucho.

Bettcher, K.J., J.E. Valdez y L.M. Valdez

1999 Salvage excavation of a Wari burial chamber at Seqllas, Ayacucho Valley, Peru. Ponencia presentada al 18th Annual Northeast Conference on Andean Archaeology and Ethnohistory, University of Massachusetts, Amhers.

Blom, D.E., B. Hallgrimsson, L. Keng, M.C. Lozada y J.E. Buikstra

1998 Tiwanaku "colonization": Bioarchaeological implications for migration in the Moquegua Valley, Peru. World Archaeology 30:238-261.

Buikstra, J.E. y D.H. Ubelaker

1994 Standards for Data Collection from Human Skeletal Remains. Proceedings of a Seminar at The Field Museum of Natural History. Arkansas Archaeological Survey Research Series № 44. Fayetteville, Arkansas.

Carmichael, P.H.

1988 Nasca Mortuary Customs: Death and Ancient Society on the South Coast of Peru. Ph.D. Dissertation, Department of Archaeology, University of Calgary, Calgary.

1995 Nasca burial patterns: Social structure and mortuary ideology. En Tombs for the Living: Andean Mortuary Practices, editado por T.D. Dillehay, pp. 161-187. Dumbarton Oaks, Washington, D.C.

Cook, A.G.

2001 Huari D-shaped structures, sacrificial offerings, and divine rulership. En Ritual Sacrifice in Ancient Peru, editado por E.P. Benson y A.G. Cook, pp. 137-163. University of Texas Press, Austin.

Donnan, C.B. y G. Cook

1997 The Pacatnamú Papers, Vol. II. Fowler Museum of Cultural History, Los Angeles.

Donnan, C.B. y C.J. Mackey

1978 Ancient Burial Patterns of the Moche Valley, Peru. University of Texas Press, Austin.

Hampe, T.

1982 Momias de los Incas en Lima. Revista del Museo Nacional 46:507-543.

Hoshower, L.M., J.E. Buikstra, P.S. Goldstein y A. Webster 1995 Artificial cranial deformation at the Omo M10 site: A
Tiwanaku complex from the Moquegua Valley, Peru. Latin American Antiquity 6:145-164.

Indriate, E. y J.E. Buikstra

2001 Coca chewing in prehistoric coastal Peru: Dental evidence. American Journal of Physical Anthropology 114:242-257.

Isbell, W.H.

1988 City and state in Middle Horizon Huari. En Peruvian Archaeology, editado por R.W. Keatinge, pp. 164-189. Cambridge University Press, Cambridge.

1997a Reconstructing Huari: A cultural chronology for the capital city. En Emergence and Change in Early Urban Societies, editado por L. Manzanilla, pp. 181-227. Plenum Press, New York.

1997b Mummies and Mortuary Monuments: A Postprocessual Prehistory of Central Andean Organization. University of Texas Press, Austin.

2001 Repensando el Horizonte Medio: el caso de Conchopata, Ayacucho, Perú. Boletín de Arqueología PUCP 4:9-68.

2004 Mortuary preferences: A Wari culture case study from Middle Horizon Peru. Latin American Antiquity 15:3-32.

Isbell, W.H., C. Brewster-Wray y L.E. Spickard

1991 Architecture and spatial organization at Huari. En Huari Administrative Structure: Prehistoric Monumental Architecture and State Government, editado por W.H. Isbell y G.F. McEwan, pp. 19-55. Dumbarton Oaks, Washington, D.C.

Isbell, W.H. y A. Cook

1987 Ideological origins of an Andean conquest state. Archaeology 40:27-33.

2002 A new perspective on Conchopata and the Andean Middle Horizon. En Andean Archaeology II: Art, Landscape, and Society, editado por H. Silverman y W.H. Isbell, pp. 249305. Kluwer Academic \& Plenum Publishers, New York.

Isbell, W.H. y K.J. Schreiber

1978 Was Huari a state? American Antiquity 43:372-389.

Isla, J.

2001 Wari en Palpa y Nasca: perspectivas desde el punto de vista funerario. Boletín de Arqueología PUCP 5:555-584.

Kent, J. y M. Kowta

1994 The cemetery at Tambo Viejo, Acari Valley, Peru. Andean Past 4:109-140.

Krogman, W.M. y M.Y. Iscan

1986 The Human Skeleton in Forensic Medicine. Charles C. Thomas, Springfield.

Lozada, M.C. y J.E. Buikstra

2002 El Señorío de Chiribaya en la Costa Sur del Perú. Instituto de Estudios Peruanos, Lima.

Lumbreras, L.G.

1974 Las Fundaciones de Huamanga: Hacia una Prehistoria de Ayacucho. Editorial Nueva Educación, Lima.

Machaca, N.G.

1996 Secuencia Cultural y Nuevas Evidencias de Formación Cultural en Ñawimpukio. Trabajo de Investigación presentado para optar la Licenciatura en Arqueología. Facultad de Ciencias Sociales, Universidad de Huamanga, Ayacucho. 
Menzel, D.

1964 Style and time in the Middle Horizon. Nawpa Pacha 2:1-106.

Morell, V.

2002 Empires across the Andes. National Geographic Magazine 206(6):106-129.

Nelson, A. y L.J. Castillo

1997 Huesos a la deriva: tafonomía y tratamiento funerario en entierros Mochica tardío de San José de Moro. En La Muerte en el Antiguo Perú: Contextos y Conceptos Funerarios, editado por P. Kaulicke, pp. 137-163. Pontificia Universidad Católica del Perú, Lima.

Ochatoma, J. y M. Cabrera

2001 Poblados Rurales Huari: Una Perspectiva desde Aqo Wayqo. Ediciones Lluvia, Lima.

2002 Religious ideology and military organization in the iconography of a D-shaped ceremonial precinct at Conchopata. En Andean Archaeology II: Art, Landscape, and Society, editado por H. Silverman y W.H. Isbell, pp. 225-247. Kluwer Academic \& Plenum Publishers, New York.

Pizarro, P.

1965 [1571] Relación del descubrimiento de los reinos del Perú. En Crónicas del Perú V, pp. 159-242. Biblioteca de Autores Españoles, Tomo CLXVIII. Ediciones Atlas, Madrid.

Pozzi-Escot, D.

1985 Conchopata, un poblado de especialistas durante el Horizonte Medio. Bulletin de L'Institut Français d' Etudes Andines 14:115-129.

1991 Conchopata: A community of potters. En Huari Administrative Structure: Prehistoric Monumental Architecture and State Government, editado por W.H. Isbell y G.F. McEwan, pp. 81-92. Dumbarton Oaks, Washington, D.C.

Rowe, J.H.

1946 Inca culture at the time of the Spanish conquest. En Handbook of South American Indians. Vol. 2: The Andean Civilizations, editado por J.H. Steward, pp. 183-330. Smithsonian Institution Bureau of American Ethnology Bulletin № 143 . Washington, D.C.

Schreiber, K.J.

1992 Wari Imperialism in Middle Horizon Peru. Anthropological Papers № 87, Museum of Anthropology, University of Michigan, Ann Arbor.

Sillar, B.

1992 The social life of the Andean dead. Archaeological Review of Cambridge 11:107-124.

Silverman, $\mathrm{H}$.

1996 Contextualizando la muerte en los cementerios Paracas. En Al Final del Camino, editado por L. Millones y M. Lemlij, pp. 1-19. Seminario Interdisciplinario de Estudios Andinos, Lima.

Stothert, K.E.

1978 Preparing a mummy bundle: Note on a late burial from Ancon, Peru. Nawpa Pacha 16:13-22.

Tung, T.A.

2003 A Bioarchaeological Perspective on Wari Imperialism in the Andes of Peru: A View from Heartland and Hinterland
Skeletal Populations. Ph.D. Dissertation, Department of Anthropology, University of North Carolina at Chapel Hill. Ubelaker, D.H.

1978 Human Skeletal Remains: Excavation, Analysis, Interpretation. Manuals on Archaeology № 2. Taraxacum, Washington.

Valdez, L.M.

2002 Marayniyoq: evidencias de producción de chicha en un establecimiento Wari. Gaceta Arqueológica Andina 26:69-86.

2006 Maize beer production during Middle Horizon Peru. Journal of Anthropological Research 62:53-80.

Valdez, L.M., K.J. Bettcher y J.E. Valdez

2000 Una Cámara Funeraria en Seqllas, Ayacucho. Boletín del Museo de Arqueología y Antropología 3:2-7.

2002a New Wari mortuary structures in the Ayacucho Valley, Peru. Journal of Anthropological Research 58:389-497.

2002b Investigaciones arqueológicas en Marayniyoq, Ayacucho: informe de la segunda temporada. Boletín del Museo de Arqueología y Antropología 5:31-42.

Valdez, L.M., K.J. Bettcher, J.A. Ochatoma, y J.E. Valdez 2005 Mortuary preferences and selected references: A comment on Middle Horizon Wari burials. Manuscrito en posesión de los autores.

Valdez, L.M., J.E. Valdez y K.J. Bettcher

2001 Posoqoypata, un cementerio Wari en el valle de Ayacucho, Perú. Bulletin de L'Institut Français d'Etudes Andines 30:335-357.

2005 Prácticas funerarias Wari del valle de Ayacucho, Perú. Corriente Arqueológica 1:173-198.

Valdez, L.M., J.E. Valdez, K.J. Bettcher y C. Vivanco 2000 Marayniyoq, un establecimiento Wari en el valle de Ayacucho, Perú. Boletín de Arqueología PUCP 4:549-564.

Verano, J.W.

1995 Where do they rest? The treatment of human offerings and trophies in Ancient Peru. En Tombs for the Living: Andean Mortuary Practices, editado por T.D. Dillehay, pp. 189-227. Dumbarton Oaks, Washington, D.C.

1997 Human skeletal remains from Tomb 1, Sipán (Lambayeque river valley, Peru) and their social implications. Antiquity 71:670-682.

2001 The physical evidence of human sacrifice in ancient Peru. En Ritual Sacrifice in Ancient Peru, editado por E.P. Benson y A.G. Cook, pp. 165-184. University of Texas Press, Austin.

2003 Human skeletal remains from Machu Picchu: A reexamination of the Yale Peabody Museum's collections. En The 1912 Yale Peruvian Scientific Expedition Collections from Machu Picchu: Human and Animal Remains, editado por L.C. Salazar y R.L. Burger, pp. 65-117.Vol. 85 Peabody Museum of natural History, New Haven.

Zapata Rodríguez, J.

1997 Arquitectura y contextos funerarios Wari en Batán Urdu, Cusco. Boletín de Arqueología PUCP 1:165-206. 
